
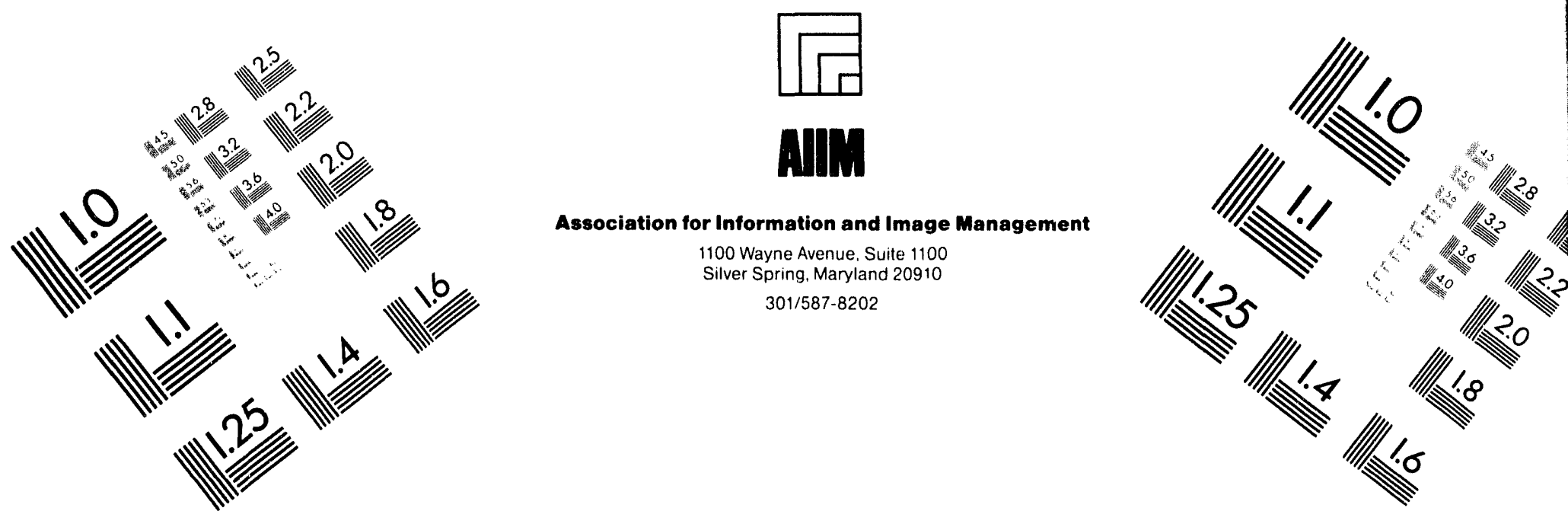

Centimeter

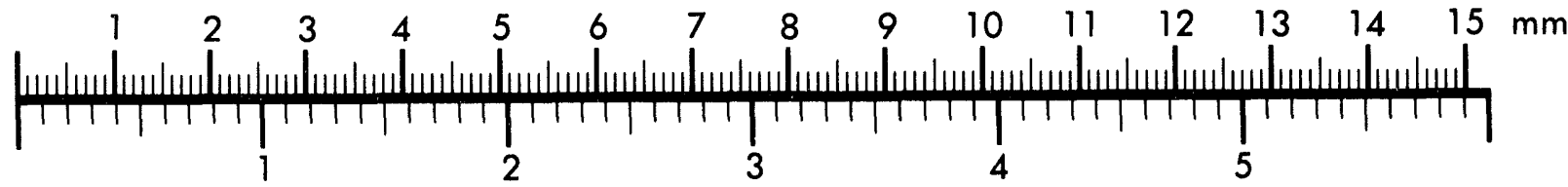

Inches
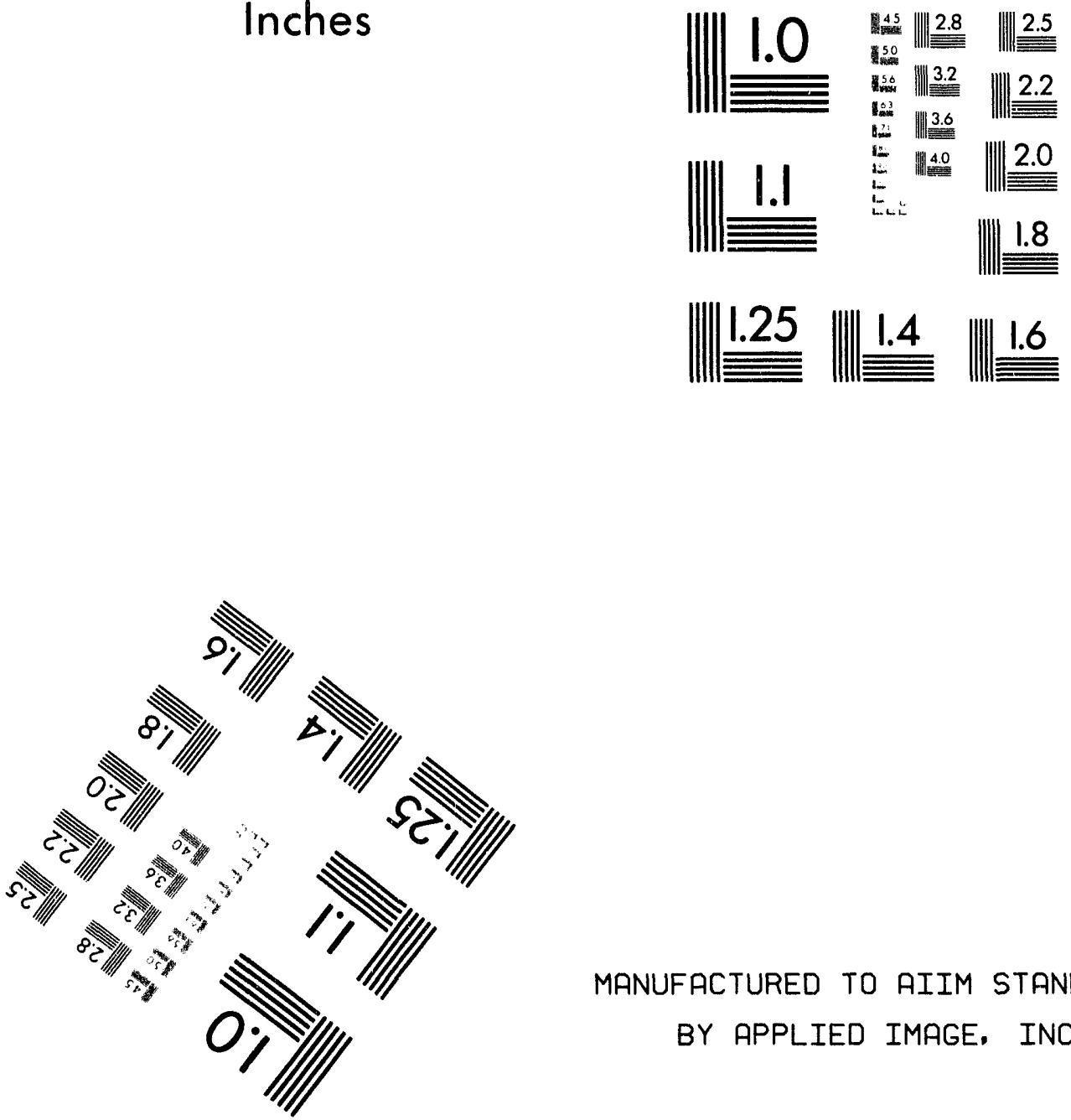

MANUFACTURED TO AIIM STANDARDS

BY APPLIED IMAGE, INC.

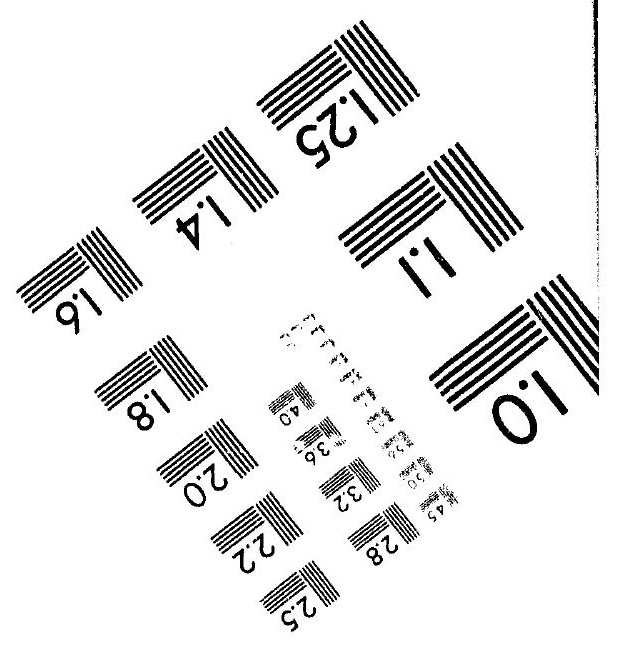



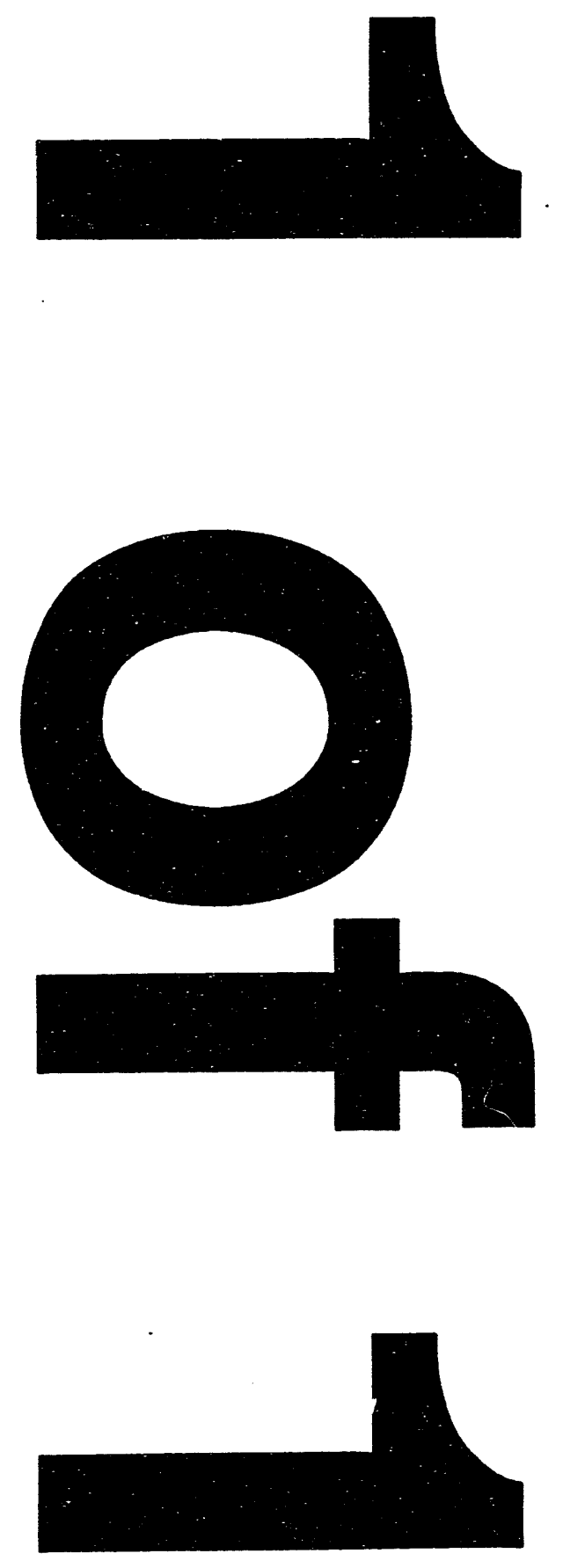
LBL - 35438

\title{
Stellar Alchemy: The Origin of the Chemical Elements
}

\author{
Eric B. Norman
}

Nuclear Science Division, Lawrence Berkeley Laboratory University of California, Berkeley, California 94720, USA

March 13, 1994

This work was supported by the Director, Office of Energy Research Division of Nuclear Physics of the Office of High Energy and Nuclear Physics of the U.S. Department of Energy under Contract DE-AC03-76SF00098

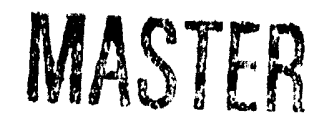




\title{
Stellar Alchemy: The Origin of the Chemical Elements
}

Eric B. Norman

Nuclear Science Division

Lawrence Berkeley Laboratory

Berkeley, CA 94720

\begin{abstract}
What makes the stars shine? This question puzzled human beings for thousands of years. Early in this century, chemists and physicists discovered radioactivity; and the nuclear model of the atom was developed. Once nuclear reactions were produced in the laboratory, it did not take long before their role in stellar energy generation was realized. The theory that nuclear fusion is the source of stellar energy was initially developed in the 1930's and was elaborated in detail in the 1950's. Only within the last ten years, however, have astronomical observations provided direct confirmation of these theoretical ideas. In this paper, I describe the sequences of nuclear reactions that are believed to be responsible for the power generation in stars. The ashes of these reactions are the heavy elements that we find on earth and throughout the universe. The evolution and final fates of stars are examined. The key astronomical observations that provide support for these theoretical ideas are presented.
\end{abstract}


Introduction

By examining the light that con $e^{t}$ to us from the stars, it is possible to deduce a great cieal of information about the nature of the universe. Remarkably, it seems that the same basic laws of physics and chemistry apply everywhere we look. Furthermore, all of the astronomical objects that we can observe seem to be made up of the same $\sim 92$ chemical elements found on Earth. From such observations, we now know that approximately $73 \%$ of the mass of the visible universe is in the form of hydrogen. Helium makes up about $25 \%$ of the mass, and everything else represents only $2 \%$ of the mass of the universe. While the abundance of these "heavy" ( $A>4$ ) elements seems quite low, it is important to remember that most of the atoms in our bodies and in the Earth are a part of this small portion of the matter in the universe. It is generally believed that the hydrogen and helium were produced in the hot, dense conditions of the birth of our universe known as the Big Bang. As will be discussed below, the heavy elements are the products of nuclear reactions in stars. Several excellent books have been written on this subject of nuclear astrophysics $(1,2,3)$, and I have relied heavily on them in preparing this talk.

While the notion that nuclear fusion reactions are the source of stellar energies is now generally agreed upon, until the last ten years or so, this conclusion was based almost entirely on circumstantial evidence. The reason for this is quite simple. The light we observe from stars is emitted from the surface; thus we cannot look inside to determine what is actually going on. We must rely on more indirect means, or use sensors that are sensitive to other types of radiation to extend our "vision". One of the early pieces of evidence that nuclear reactions do occur in stars was the observation of spectral lines of the element technetium on the surfaces of certain old stars. Technetium is one of only two elements below bismuth that has no stable isotopes. In fact, the isotope believed to be observed in stars, ${ }^{99} \mathrm{Tc}$, has a half-life of only $2 \times 10^{5}$ years. While this may seem long by human standards, it is very short on astronomical timescales. The only plausible way for such "short-lived" material to be present in a star is for it to have been recently synthesized within that star. 
Another important piece of astronomical data is the observed relationship between the surface temperatures and luminosities of stars. Shown in Figure 1 is what is known as a Hertzsprung-Russell (HR) diagram. It has been found that about $80 \%$ of all the observed stars, including our Sun, fall on a roughly diagonal band known as the main sequence. There are also two other important classes of stars. In the upper right-hand corner of the HR diagram, there is a group of cool but luminous stars known as red giants. In the lower left-hand corner, there is a population of hot but dim stars known as white dwarfs. The significance and origin of these stellar classes will be discussed later in terms of stellar evolution.

Finally, a large amount of information can be obtained from more detailed analysis of the elemental and isotopic composition of matter. Shown in Figure 2 are the observed abundances of the material in our solar system. As discussed previously, hydrogen and helium are by far the most abundant species. The next heaviest group of elements, $\mathrm{Li}, \mathrm{Be}$, and $\mathrm{B}$, are by comparison exceedingly rare. Above this group, the abundances start out higher, but gradually decrease as one moves up to heavier eilements. Several important features about this pattern should be pointed out. There is a large abundance peak near mass 60 that is associated with the elements around iron. Above this point, there is again a general decrease in abundance with increasing mass number that is interrupted by two double-peaked structures. Distinct abundance peaks are observed around mass numbers $130,140,195,208$. Taken as a whole, this abundance distribution provides many clues to the source of stellar energies and the origin of the chemical elements.

\section{What Makes the Stars Shine?}

One of the most basic questions that we can ask about stars is the source of their energies, or in other words, what makes the stars shine? Before one can answer this question, some basic properties of stars must be known. Taking our Sun as a representative star, we know that:

$$
\begin{aligned}
& M_{\Theta}=2 \times 10^{33} \text { grams } \\
& L_{\Theta}=4 \times 10^{33} \mathrm{ergs} / \text { second }
\end{aligned}
$$




$$
A_{\Theta}=4.5 \times 10^{9} \text { years, }
$$

where $\mathrm{M}_{\odot}, \mathrm{L}_{\odot}$, and $\mathrm{A}_{\odot}$ are the mass, luminosity, and age of the Sun, respectively. We know that life has existed on Earth for at least the last $2 \times 10^{9}$ years, and this requires that the Sun's luminosity not have changed dramatically over that period of time. Therefore, over the lifetime of the Sun, it is reasonable to assume that it has radiated a total of $L_{\Theta} A_{\Theta}=6 \times 10^{50} \mathrm{ergs}$, or $3 \times 10^{17} \mathrm{ergs} / \mathrm{gram}$.

There are several mechanisms that have been considered as possible sources of this energy. Exothermic chemical reactions are responsible for much of the energy generation on Earth. Perhaps that's what powers the stars. However, the maximum energy release in such reactions is approximately $2 \times 10^{12} \mathrm{ergs} / \mathrm{gram}$. Chemical reactions could therefore maintain the Sun at its present luminosity for only about 30,000 years. Thus, chemical reactions cannot be the source of stellar energies.

Gravitation is another possible energy source. If one assumes that the matter in the Sun contracted from infinite initial separation down to the present radius, then the change in gravitational potential energy could be the source of the Sun's energy. However, one can easily calculate that the total gravitational potential energy of the Sun (assuming constant density throughout) is

$$
\mathrm{V}=3 / 5\left(\mathrm{GM}^{2} / \mathrm{R}\right)=2 \times 10^{48} \mathrm{ergs} .
$$

This translates to about $1015 \mathrm{ergs} / \mathrm{gram}$, but is still far short of what is required.

Near the beginning of this century it was discovered that nuclear reactions are capable of producing large amounts of energy, and the possible role of nuclear reactions in stars was soon realized. Consider, for example, combining four hydrogen nuclei in such a way as to produce a nucleus of ${ }^{4} \mathrm{He}$. It turns out that the total mass of four hydrogen nuclei is a bit larger than that of one ${ }^{4} \mathrm{He}$ nucleus. According to Einstein's famous equation, $\mathrm{E}=\mathrm{mc}^{2}$. This implies that if one could completely convert 1 gram of matter into energy, $9 \times 10^{20}$ ergs would be released. Thus, the fusion of hydrogen into helium is an exothermic reaction due to the conversion of mass into energy. We will see later on that this does not actually occur in one step, but in fact requires a number of separate reactions. However, all that matters for the present discussion is the fact that 
for each ${ }^{4} \mathrm{He}$ nucleus produced in this way, $\mathrm{Q}=26 \mathrm{MeV}$ is released. Recalling that $1 \mathrm{MeV}=$ $1.602 \times 10^{-6} \mathrm{erg}$, one can now calculate the energy generation efficiency of this process,

$$
\varepsilon=\mathrm{Q} / 4 \mathrm{~m}_{\mathrm{p}} \mathrm{c}^{2}=7 \times 10^{-3},
$$

where $m_{p}=$ mass of a hydrogen nucleus $=1.67 \times 10^{-24} \mathrm{gram}$, and $\mathrm{c}=$ the speed of light $=3 \times 10^{10}$ $\mathrm{cm} / \mathrm{sec}$. Thus the fusion of hydrogen into helium yields

$$
\varepsilon\left(9 \times 10^{20} \mathrm{ergs} / \mathrm{gram}\right)=6.3 \times 10^{18} \mathrm{ergs} / \mathrm{gram} .
$$

Therefore, only about $5 \%$ of the hydrogen in the Sun need be "burned" into helium in order to meet the energy generation requirement. Thus it has been concluded that the sources of stellar energies are nuclear fusion reactions. We shall also see that the "ashes" of these reactions are the elements between carbon and iron.

Figure 3 illustrates the potential of two nuclei as a function of their separation. At large distances, they repel one another via the long range Coulomb force, while at short distances the strong, attractive nuclear force takes over. In order for a nuclear reaction to occur, the two nuclei must reach a separation approximately equal to the sum of their radii. The energy required to bring two nuclei with electric charges $Z_{1}$ and $Z_{2}$ and masses $A_{1}$ and $A_{2}$ to this point can easily be calculated, and is known as the Coulomb barrier.

$$
E_{c} \approx\left(Z_{1} Z_{2}\right) /\left(A_{1}^{1 / 3}+A_{2}^{1 / 3}\right) M e V .
$$

As an example, consider the interaction of two hydrogen nuclei, which we will see is the first step in the synthesis of helium from hydrogen. The Coulomb barrier for this reaction is about 0.5 MeV. This must be compared with the typical thermal energies found in stars. At the center of our Sun, the temperature, $T_{0}$, is approximately $15 \times 10^{6} \mathrm{~K}$. Thus, the mean thermal kinetic energy of a nucleus at the center of the Sun is $3 / 2 \mathrm{kT} \mathrm{T}_{\mathrm{o}}=2 \mathrm{keV}$. Under such conditions, classical physics says that the two hydrogen nuclei can never get close enough together for a nuclear reaction to occur. Nevertheless, the stars shine! The nuclear reactions that power the stars proceed via quantum mechanical tunneling through the potential barrier. I would point out that this is a general feature of all of the major nuclear burning stages of stars. During helium burning, which occurs at a temperature of about one hundred million degrees, the mean thermal energy is about $13 \mathrm{keV}$. Even 
at a temperature of one billion degrees, which is appropriate for oxygen burning, the thermal energy is only $130 \mathrm{keV}$, while the Coulomb barrier between two oxygen nuclei is on the order of $12 \mathrm{MeV}$.

Now let's examine more closely the sequence of reactions that are believed to be occurring in our Sun. The energy generated by the fusion of hydrogen into helium provides main sequence stars their support against gravitational contraction. The detailed sequences of nuclear reactions responsible for hydrogen burning in our Sun. and in other stars was worked out in the 1930's by Bethe, Critchfield, and von Weizsacker $(4,5,6,7)$. Shown in Figure 4 are the reactions and the percentage of the time each occurs in the Sun. The first reaction involves the "weak" interaction and is the rate-determining step in the sequence. The mean lifetime against this reaction for a hydrogen nucleus at the center of the Sun is about ten billion years. This makes direct laboratory studies of this reaction impossible, but ensures the long lifetime of stars such as our Sun. As mentioned above, we cannot directly see what is actually going inside the Sun. A photon produced at the center of the Sun scatters many times and loses memory of its nuclear origin as it works its way outward. In fact, it takes about $10^{7}$ years for such a photon to travel to the surface. However, there is another type of radiation produced during the conversion of hydrogen into helium which interacts so feebly with matter that it is able to freely escape from the center of the Sun. This object is the neutrino, and by building detectors which can "see" neutrinos, we can learn much about what really goes on inside a star.

O.e can easily estimate $\Phi_{v}$, the flux of solar neutrinos at the Earth's surface. Assuming that the present observed luminosity of the Sun is produced by the fusion of hydrogen into helium, then

$$
\Phi_{v}=2 \mathrm{~L}_{\Theta} \mathrm{Q},
$$

where $\mathrm{L}_{\Theta}=1 \mathrm{kilowatt} / \mathrm{m}^{2}$ (the power of sunlight at the Earth's surface) and $\mathrm{Q}=26 \mathrm{MeV}$ the energy released in the fusion of four hydrogen atoms into one helium atom). One thus obtains

$$
\Phi_{v}=5 \times 1010 / \mathrm{cm}^{2}-\mathrm{sec} \text {. }
$$


This implies that about 250 trillion neutrinos are going through each of us every second! We don't see them, feel them, or smell them, but they are there. How do we know? Well, there are now four working solar neutrino detectors in the world. Three are radiochemical experiments - one based on the ${ }^{37} \mathrm{Cl}-{ }^{37} \mathrm{Ar}$ system and two based on the ${ }^{71} \mathrm{Ga}-{ }^{71} \mathrm{Ge}$ system. However, the first experiment to provide conclusive direct evidence of solar neutrinos was the Kamiokande II water Cerenkov counter ( 8 ). This experiment used a large tank of water located deep underground in a lead mine in Japan to detect the interactions of solar neutrinos with electrons. The incoming solar neutrinos occasionally scatter elastically off of atomic electrons. The electrons tend to recoil in the direction in which the incoming neutrino was traveiling. When one of these electrons travels faster than the speed of light in water ( which does not violate the laws of Special Relativity !), an electromagnetic "shock wave" known as Cerenkov radiation is produced. This is analogous to the sonic boom produced by supersonic aircraft. The Cerenkov radiation is the eerie blue glow that can be seen in nuclear reactor cooling pools. By surronding the water tank with a large number of photomultiplier tubes, one can measure the amount and pattern of Cerenkov light produced in these interactions. From this information, the incoming neutrino's energy and direction can be reconstructed. Figure 5 illustrates the results of nearly three years of operation of this experiment. What is shown is the number of events observed in this counter versus the cosine of the angle between the neutrino's incoming direction and the position of the Sun. The strong forward peaking of this distribution conclusively proves that the Sun was the origin of these neutrinos. Thus for the first time we were able to "look" inside the Sun and see direct proof that the origin of the Sun's luminosity is nuclear fusion. It should be pointed out, however, that all four of the experiments done to date show a deficit of solar neutrinos compared to the theoretical expectations. Thus there may still be some surprises to come in the area of solar neutrino astronomy.

A star will burn hydrogen into helium until the hydrogen in the core is exhausted. At this point, the star begins to move off the main sequence and becomes a red giant. Because the energy generation mechanism is turned off, the core of the star contracts. The central temperature rises until helium is "ignited." A quick look at a nuclear physics text book will reveal that there are no 
particle-stable nuclei with either mass 5 or 8 . Thus helium burning cannot proceed via two-body reactions such as ${ }^{4} \mathrm{He}+{ }^{1} \mathrm{H}$ or ${ }^{4} \mathrm{He}+{ }^{4} \mathrm{He}$. From Figure 2 it can be seen that following hydrogen and helium, carbon and oxygen are the two most abundant elements in the universe. The question is how were they produced?

The answer was provided by E. Salpeter, E. Opik, and F. Hoyle $(9,10,11,12,13)$ who realized that in order to bridge the stability gaps at $\mathrm{A}=5$ and 8 , a "three-body" reaction was necessary. As can be seen in Figure 6, a ${ }^{8} \mathrm{Be}$ nucleus is a bit heavier than two ${ }^{4} \mathrm{He}$ nuclei. As a result, ${ }^{8} \mathrm{Be}$ decays into two ${ }^{4} \mathrm{He}$ nuclei with a lifetime of $2 \times 10^{-16}$ seconds. This means that at the temperatures and densities appropriate for helium burning, an equilibrium can be established between ${ }^{4} \mathrm{He}$ and ${ }^{8} \mathrm{Be}$. At a temperature of $10^{8} \mathrm{~K}$ and a density of $10^{5} \mathrm{gram} / \mathrm{cm}^{3}$,

$$
\left[{ }^{8} \mathrm{Be}\right] /\left[^{4} \mathrm{He}\right]=10^{-9} \text {. }
$$

During the brief period of its existence, ${ }^{8} \mathrm{Be}$ can capture another ${ }^{4} \mathrm{He}$ nucleus to produce ${ }^{12} \mathrm{C}$. It was soon realized that the rate for this process would be unacceptably low unless there were a suitable state in ${ }^{12} \mathrm{C}$ that could serve as a "resonance" for the ${ }^{8} \mathrm{Be}+{ }^{4} \mathrm{He}$ reaction. To be such a resonance, the state must have angular momentum equal zero, even parity, must lie close to the ${ }^{8} \mathrm{Be}$ $+{ }^{4} \mathrm{He}$ threshold energy, and have a reasonable gamma-decay branch to the ground state of ${ }^{12} \mathrm{C}$. The need for such a level was suggested by F. Hoyle in 1954, and subsequent experiments demonstrated that the right sort of level exists in ${ }^{12} \mathrm{C}$ at just the right energy to make this so-called "triple alpha-process" work. Once ${ }^{12} \mathrm{C}$ is formed; ${ }^{16} \mathrm{O}$ can be produced via the ${ }^{12} \mathrm{C}\left({ }^{4} \mathrm{He}, \gamma\right)$ reaction.

Helium burning proceeds in the stellar core until all of the helium is converted into ${ }^{12} \mathrm{C}$ and 160. After this point is reached there are two possibilities for the star. If it is sufficiently massive $\left(M \gtrsim 10 M_{\Theta}\right)$ the core will again contract, the temperature will rise, and when the central temperature reaches about $5 \times 10^{8} \mathrm{~K}$, carbon will "ignite". On the other hand, in low-mass stars the core temperatue never gets high enough to burn carbon. Therefore, no further energy-generating reactions are possible. Such stars quietly end their lives as white dwarfs with their support against gravitational collapse provided by electron degeneracy pressure. In the 1930's, Chrandrasekhar 
(14) showed that the maximum stellar mass that can be supported in this way is $1.4 \mathrm{M}_{\odot}$. More massive stars cannot end their lives in this way and are destined to become either neutron stars or black holes.

For the more massive stars, once the core temperature reaches $5 \times 10^{8} \mathrm{~K}$, the ${ }^{12} \mathrm{C}+{ }^{12} \mathrm{C}$ reaction produces large amounts of ${ }^{20} \mathrm{Ne}$ and ${ }^{24} \mathrm{Mg}$. At $1 \times 10^{9} \mathrm{~K}$, oxygen burning begins and $16 \mathrm{O}$ $+{ }^{16} \mathrm{O}$ reactions produce ${ }^{28} \mathrm{Si}$ and ${ }^{32} \mathrm{~S}$. The synthesis of still heavier elements does not proceed directly through reactions such as ${ }^{28} \mathrm{Si}+{ }^{28} \mathrm{Si}$, because in order to overcome the high Coulomb barriers, temperatures on the order of $4.5 \times 10^{9} \mathrm{~K}$ are required. At such high temperatures, photodisintegration reactions become important which allows the following sort of rearrangement reactions to occur:

$$
\begin{aligned}
& { }^{28} \mathrm{Si}+\gamma \rightarrow{ }^{24} \mathrm{Mg}+{ }^{4} \mathrm{He} \\
& { }^{28} \mathrm{Si}+{ }^{4} \mathrm{He} \rightarrow{ }^{32} \mathrm{~S}+\gamma .
\end{aligned}
$$

Under these conditions, nuclear statistical equilibrium can occur which leads to the synthesis of the most tightly bound nuclei - "iron peak" elements. In particular, it is expected that large amounts of the radioactive isotope ${ }^{56} \mathrm{Ni}\left(\mathrm{t}_{1 / 2}=6\right.$ days) are produced through this sequence of reactions.

The time that a massive star spends in each of these burning stages gets progressively shorter as the star evolves. Shown in Table 1 are the results of calculations of the evolution of a star whose mass is 25 times that of our Sun (15). While such a star spends millions of years in its initial hydrogen burning stage, it spends only about one day in its final silicon burning stage.

Once the core of the star is converted into iron-group nuclei, the star has nearly reached the end of its life. Because the binding energy per nucleon reaches a maximum at this point, there are no further energy generating reactions possible. Thus, once again the core of the star will start to contract and heat up. Eventually the point of iron photodisintegartion is reached. This energy drain further removes support against gravitational collapse. The details of what happens next are not entirely clear, but we know what the final result is - a supernova explosion. If one could look inside such a star just prior to the explosion, it is believed that it would exhibit the "onion-skin" 
structure illustrated in Figure 7. The deeper inside the star one looks, the higher is the peak temperature and correspondingly the heavier are the nuclei that are synthesized.

As the collapse of the core occurs, the density grows to the point where it becomes energetically favorable for electrons to be captured by protons, producing neutrons and neutrinos. This neutronizes the core of the star and produces a huge burst of neutrinos. Eventually, the core reaches and then exceeds the density of nuclear matter. At this point the equation of state of the matter stiffens, and a hydrodynamic bounce occurs. Through the scattering of the neutrinos, or the bounce, or some combination of the two, a supernova explosion occurs in which the mantle of the star is blown off, leaving behind a neutronized remnant. If the mass of the remnant is less than 2$3 \mathrm{M}_{\odot}$, it will settle down as a neutron star supported against further collapse by the pressure of degenerate neutrons. More massive stars are believed to continue to collapse and form black holes. The ashes of these sequences of first static and then explosive nuclear reactions are the bulk of the elements between carbon and iron.

A supernova is an extremely violent and catastrophic event in which the bulk of a star is dismantled in a very short period of time. It produces a brilliant display of energy as the mantle of the star is heated up and expands. But as awesome as the optical observations appear, it is important to realize that the light show that is observed represents only about $1 \%$ of the energy released in the supernova explosion. The other $99 \%$ of the energy is believed to be radiated away in the form of neutrinos!

This detailed theory of the evolution of massive stars was worked out long ago on the basis of accumulated circumstantial evidence. However, Nature only recently provided us with an opportunity to test the predictions of these theoretical ideas. On February 23, 1987, a blue-giant star known as SS69202 became a supernova. This supernova, later designated SN1987A, was discovered through naked-eye observations made by astronomers in Chile. This star was located in a nearby companion of our own galaxy, the Large Magellanic Cloud (LMC). The distance to the LMC is estimated to be approximately 52 kiloparsecs or about 160,000 light years, thus making this the closest supernova to appear in over 300 years. Fortunately, astronomers and physicists 
were prepared to observe many of the phenomena predicted to be produced by a supernova of this kind.

Two water Cerenkov counters were operating at the time this supernova occurred. One was the Kamiokande detector that was described previously. The second was the IrvineMichigan-Brookhaven (IMB) detector operating in a salt mine in Ohio. On the day that the supernova was first observed optically, both of these experiments detected a burst of neutrino events unlike anything ever seen before or since. As can be seen in Figure 8, the Kamiokande detector recorded 12 events in a period of 13 seconds (16). The IMB experiment detected 8 events in a period of 6 seconds (17). The inferred energies and fluxes of neutrinos agreed well with the predictions of stellar evolution theory. Furthermore, these neutrinos appear to have come from the direction of the LMC.

As previously discussed, the sequence of nuclear fusion reactions that occur inside a massive star such as SS69202 should produce large amounts of $56 \mathrm{Ni}$. The shock waves produced in the supernova explosion undoubtedly eject a.sizable fraction of this material, as well as the outer envelope of the star, into interstellar space. The debris from other supernovae have been observed to glow long after the initial explosions. It was thought that the power for this light was provided by the energy released in the decays of the radioactive isotopes $56 \mathrm{Ni}$ and its daughter $56 \mathrm{Co}\left(\mathrm{t}_{1 / 2}=77\right.$ days). The light curve, or luminosity as a function of time, of SN1987A was measured by many observers and found to decay exponentially with a half life of precisely 77 days. Futhermore, after the material had expanöed sufficiently to allow gamma rays to escape from the debris, the characteristic gamma rays emitted in the decay of ${ }^{56} \mathrm{Co}$ to ${ }^{56} \mathrm{Fe}$ at 847 and $1238 \mathrm{keV}$ were observed by a detector on the Solar Maximum Mission satellite (18). These data are illustrated in Figure 9.

Taken as a whole, the measurements of neutrinos, the light curve, and. gamma rays from SN1987A have provided remarkable confirmation that our understanding of stellar evolution is basically correct. However, one should no: get the impression that SN1987A is the only source of such data. As can be seen in Figure 10, observations inade by detectors on the High Energy 
Astronomical Observatory (HEAO 3) show a clear signal of 1809-kev gamma rays coming from the plane of our galaxy (19). This is exactly the energy of the gamma ray emitted in the decay of ${ }^{26} \mathrm{Al}$ to ${ }^{26} \mathrm{Mg}$. The half life of ${ }^{26} \mathrm{Al}$ is $7.2 \times 10^{5}$ years, which is very short on astronomical timescales. The only way for the inferred amount of ${ }^{26} \mathrm{Al}$ to be present in our galaxy is if it is being continuously synthesized in stars.

\section{Origin of the Heavy Elements $(A>56)$}

The type of nuclear reactions discussed thus far terminate at iron. As discussed previously, because the binding energy per nucleon reaches a maximum around iron, further fusion reactions between heavy nuclei are endothermic. Furthermore the Coulomb barriers for charged-particle induced reactions become prohibitively high. It was realized over thirty-five years ago that in order to account for the observed abundances of the elements above iron, neutron capture reactions are required. The two double-humped peaks seen in the Solar System abundance distribution at $A=130,140$ and $A=195,208$ appear to be correlated with the closed-shell neutron "magic numbers" 82 and 126. Based upon this observation, it was suggested by Burbidge, Burbidge, Fowler, and Hoyle (20) that two distinct types of neutron capture processes are required. In the s- or slow process, neutron captures proceed through the isotopes of a given element until a radioactive nucleus is reached. Then, because the neutron flux is so low, beta decay almost always occurs before the next neutron comes along. Thus the path of the s-process follows the line of beta stability. In contrast, during the $r$ - or rapid process the neutron flux is so high that many many neutron captures occur before beta decay happens. The path of the r-process thus lies far to the neutron rich side of beta stability. Once the r-process neutron source turns off, these neutron-rich nuclei beta decay back to produce the stable nuclei we now observe. The calculated paths of the s- and r-processes are illustrated in Figure. 11.

Laboratory experiments have shown that nuclei with neutron magic numbers have very small neutron capture cross sections and relatively long beta decay half lives. Thus once 
prorluced, such nuclei are not easily destroyed. As a.result, in both the s- and r-processes abundance peaks are produced at the points where the neutron magic numbers 82 and 126 are encountered. These shell closures are reached at about 10 units lower atomic number in the rprocess than in the s-process. This nicely accounts for the two double-humped peaks in the abundance curve that were previously discussed.

The site of the s-process is believed to be in the helium-burning zones of red giant stars. The neutrons required for the s-process are thought to be produced through the reactions

$$
{ }^{13} \mathrm{C}+{ }^{4} \mathrm{He} \rightarrow 16 \mathrm{O}+\mathrm{n}
$$

and/or

$$
{ }^{22} \mathrm{Ne}+{ }^{4} \mathrm{He} \rightarrow{ }^{25} \mathrm{Mg}+\mathrm{n} .
$$

The neutrons generated by these reactions are then captured on "seed" nuclei. Calculations show that it is not possible to reproduce the observed s-process abundances under reasonable helium burning conditions if the seed nuclei are too light. The most likely seeds are, in fact, iron group nuclei. It is important to remember that no iron is produced during hydrogen or helium burning. Thus in order for an s-process to occur in a red-giant star, these iron nuclei had to be present when the star formed. This could only be the case if the the matter from which this star formed had already been contaminated by the ashes of previous generations of stars. Thus, the s-process is a secondary process and could not have occurred in the first stars that formed in our galaxy.

The s-process terminates at ${ }^{209} \mathrm{Bi}$ because the addition of a neutron to this nucleus produces ${ }^{210} \mathrm{Bi}$ which through alpha and beta decays eventually leads back to ${ }^{206} \mathrm{~Pb}$. In order to account for the observed abundances of thorium and uranium, a way of avoiding this point of alpha instability is required. The s-process produces approximately one-half of the nuclei between iron and bismuth. In order to explain the other half of these observed abundances and to understand the origin of the actinide elements, the r-process is required. While we know that a rapid neutroncapture process occurs in Nature, our understanding of this type of nucleosynthesis is much more limited than that for the s-process. It is generally believed that during a supernova explosion, conditions of high temperature and density allow very large neutron fluxes to be produced for brief 
periods of time in regions deep inside the star. As a result, it is thought that the r-process elements are synthesized and dispersed into the inetrstellar medium by supernovae.

\section{Conclusions}

We have seen that charged-particle-induced nuclear fusion reactions are the sources of stellar energies. Main sequence stars, such as our Sun, spend most of their lives quietly converting hydrogen into helium. Later stages of stellar evolution involve fusion reactions of heavier nuclei. The ashes of these reactions are the elements between carbon and iron. The elements above iron are produced via neutron capture reactions. By building detectors that are sensitive to types of radiation that we cannot directly "see", we have uncovered a wealth of new information about the nature of our universe. Recent astronomical observations of neutrinos and gamma rays have provided dramatic confirmation that our basic ideas of stellar evolution and the

origin of the chemical elements are correct. The inevitable conclusion that can be drawn from all of this work is truly astonishing: the very atoms that make up our planet and even our bodies were synthesized billions of years ago inside the nuclear furnaces found at the centers of stars!

\section{Acknowledgement}

This work was supported by the Director, Office of Energy Research, Office of High Energy and Nuclear Physics, Nuclear Physics Division of the U. S. Department of Energy under Contract No. DE-AC03-76SF00098. 


\begin{tabular}{llll} 
Burning Stage & \multicolumn{1}{c}{ Temperature } & \multicolumn{1}{c}{ Density } & Timescale \\
\hline Hydrogen & 5 & $\left(\mathrm{geV} / \mathrm{cm}^{3}\right)$ & \\
Helium & 20 & 5 & $7 \times 10^{6}$ years \\
Carbon & 80 & 700 & $5 \times 10^{5}$ years \\
Neon & 150 & $2 \times 10^{5}$ & 600 years \\
Oxygen & 200 & $4 \times 10^{6}$ & 1 year \\
Silicon & 350 & $10^{7}$ & 6 months \\
Collapse & 600 & $3 \times 10^{7}$ & 1 day \\
Bounce & 3000 & $3 \times 10^{9}$ & seconds \\
Explosive & $100-600$ & $10^{14}$ & milliseconds \\
\hline \hline
\end{tabular}

Table 1. The major stages in the evolution of a $25 \mathrm{M}_{\Theta}$ star (Ref. 15). 


\section{Literature Cited}

1. Clayton, D. D. Principles of Stellar Evolution and Nucleosynthesis; Univ. of Chicago Press: Chicago, 1983.

2. Barnes, C. A.; Clayton, D. D.; Schramm, D. N., Eds. Essays in Nuclear Astrophysics; Cambridge Univ. Press: Cambridge, 1982.

3. Rolfs, C. E.; Rodney, W. S. Cauldrons in the Cosmos; Univ. of Chicago Press: Chicago, 1988.

4. Bethe, H. A.; Critchfield, C. L. Phys. Rev. 1938, 54, 248 and 862.

5. Bethe, H. A. Phys. Rev. 1939, 55, 103 and 434.

6. von Weizsacker, C. F. Zeit. Phys. 1937, 38, 176.

7. von Weizsacker, C. F. Zeit. Phys. 1938, 39, 633.

8. Hirata, K. S.; et al. Phys. Rev. D 1991, 44, 2241.

9. Salpeter, E. E. Phys. Rev. 1952, 88, 547.

10. Salpeter, E. E. Astrophys. J. 1952, 115, 326.

11. Opik, E. J. Proc. Roy. Irish Acad. 1951, A54, 49.

12. Hoyle, F.; Dunbar, D. N. F.; Wenzel, W. A.; Whaling, W. Phys. Rev. 1953, 92, 1095.

13. Hoyle, F. Astrophys. J. Suppl. 1954, 1, 12.

14. Chandrasekhar, S. Mon. Not. Roy. Astro. Soc. 1935, 95, 207.

15. Weaver, T. A.; Woosley, S. E. Ann. N. Y. Acad. Sci. 1980, 336, 335.

16. Hirata, K.; et al. Phys. Rev: Lett. 1987, 58, 1490.

17. Bionta, R. M.; et al. Phys. Rev. Lett. 1987, 58, 1494.

18. Matz, S. M.; et al. Nature 1988, 331, 416.

19. Mahoney, W. A.; Ling, J. C.; Wheaton, W. A.; Jacobson, A. S. Astrophys. J. 1984 286, 578.

20. Burbidge, E. M.; Burbidge, G. R.; Fowler, W. A.; Hoyle, F. Rev. Mod. Phys. 1957, $29,547$. 


\section{Figure Captions}

Fig. 1. The Hertzsprung-Russell diagram. (Ref. 3, page 44 ).

Fig. 2. Solar system abundances (by number) of the nuclides. (Ref. 2, page 23)

Fig. 3. The potential between two nuclei versus their separation. (Ref. 3, page 153).

Fig. 4. The sequences of nuclear reactions by which hydrogen in fused into helium in the Sun. (Ref. 3, page 354).

Fig. 5. Kamiokande II observation of solar neutrinos (Ref. 8).

Fig. 6. The energy-level schemes of ${ }^{8} \mathrm{Be}$ and ${ }^{12} \mathrm{C}$ and the "triple-alpha" process. (Ref. 3, page 388).

Fig. 7. Cross sectional view of pre-supernova star (Ref. 3, page 437).

Fig. 8. Kamiokande observation of neutrinos from SN1987A (Ref. 16).

Fig. 9. Solar Maximum Mission observation of ${ }^{56}$ Co gamma rays from SN1987A (Ref. 18).

Fig. 10. HEAO3 observations of ${ }^{26} \mathrm{Al}$ gamma rays from the galactic plane (Ref. 19).

Fig. 11. Calculated paths of the s- and r- neutron capture processes (Ref. 3, page 472). 


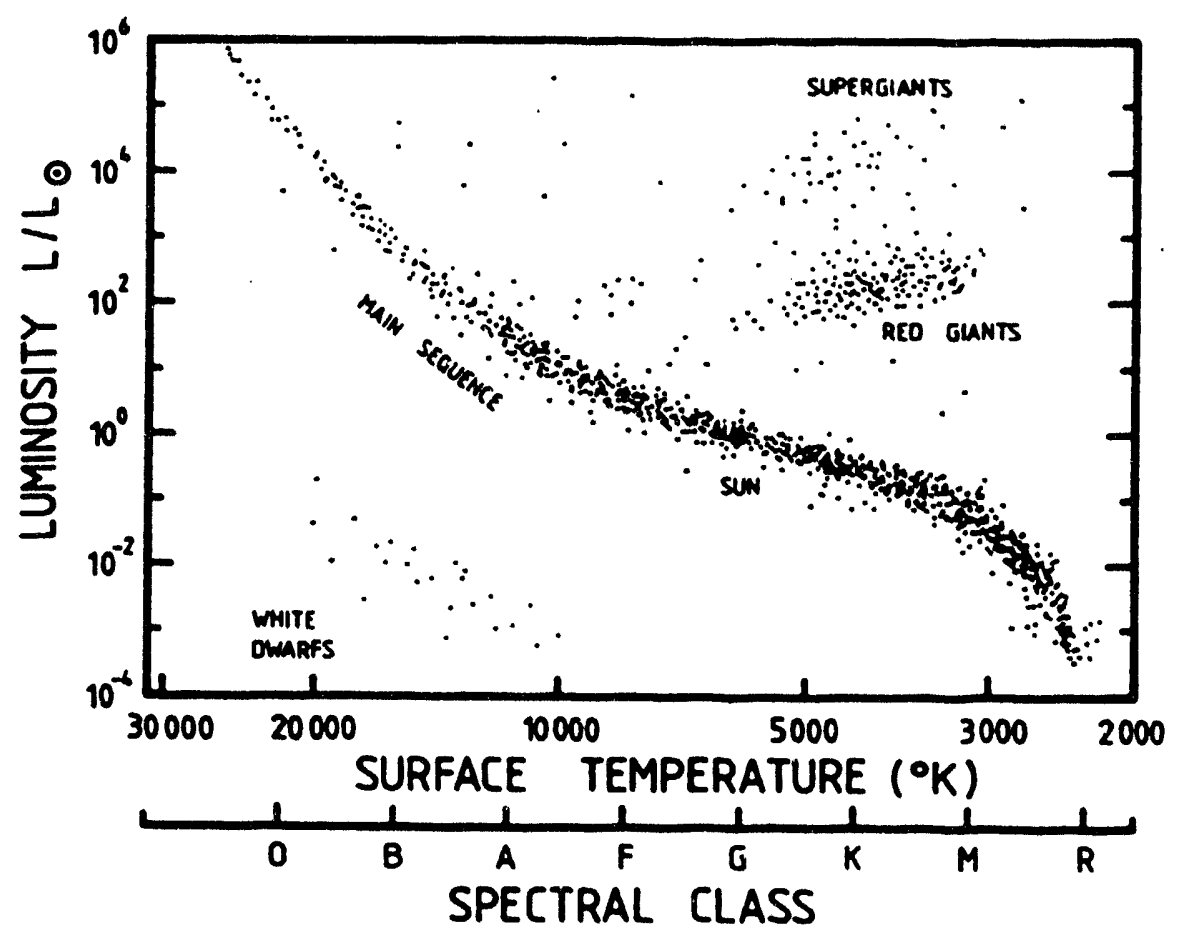

Figure 1 


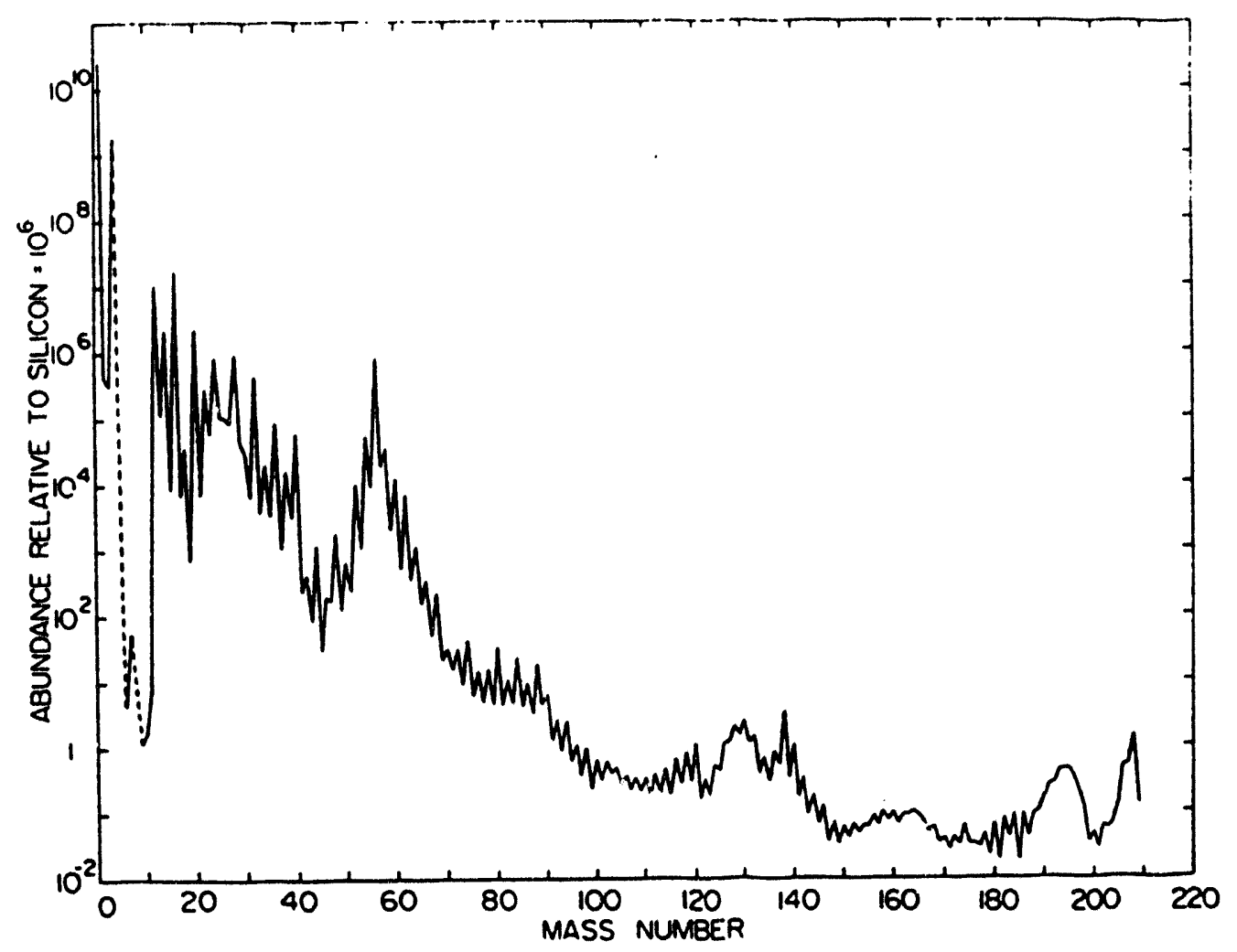

Figure 2 


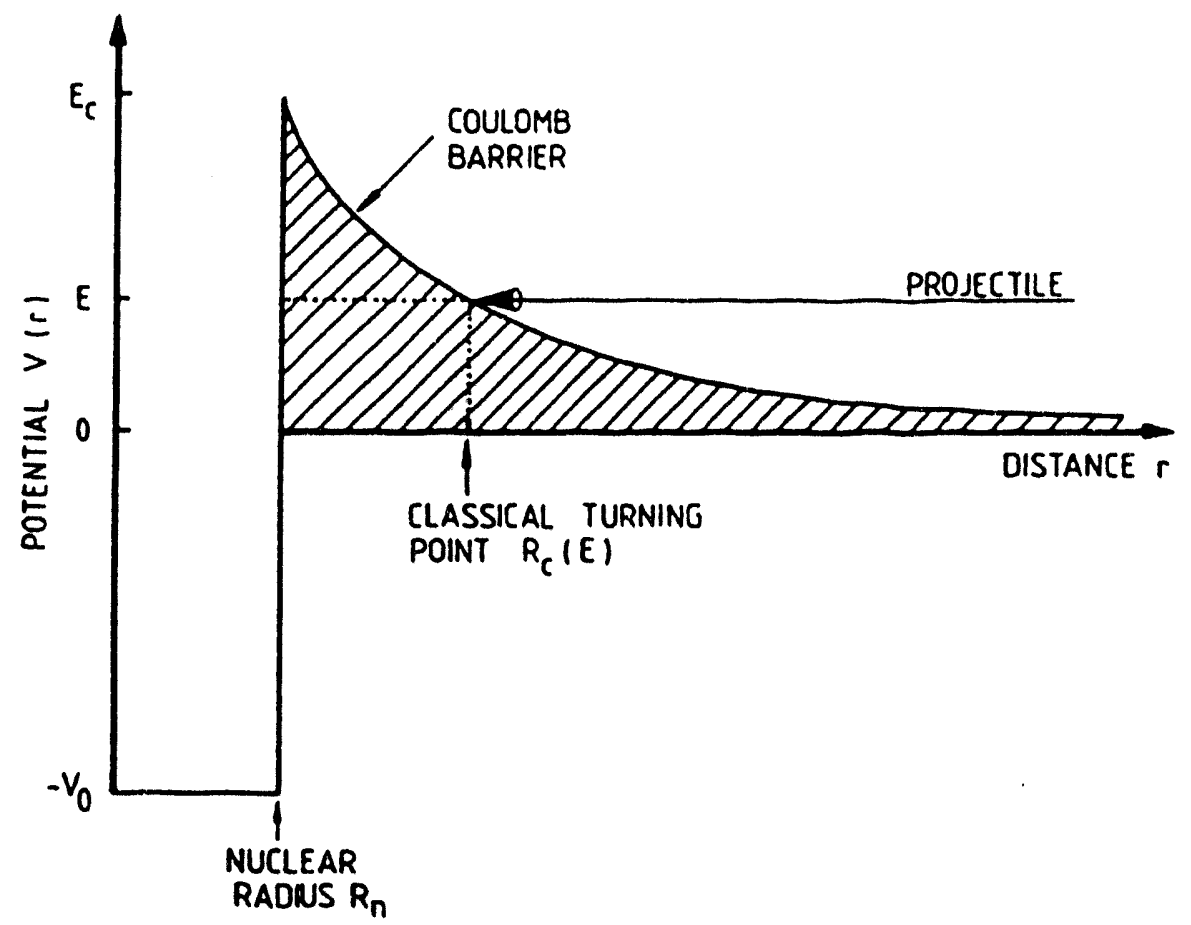

Figure 3 


\section{THE REACTIONS OF THE P-P CHAIN}

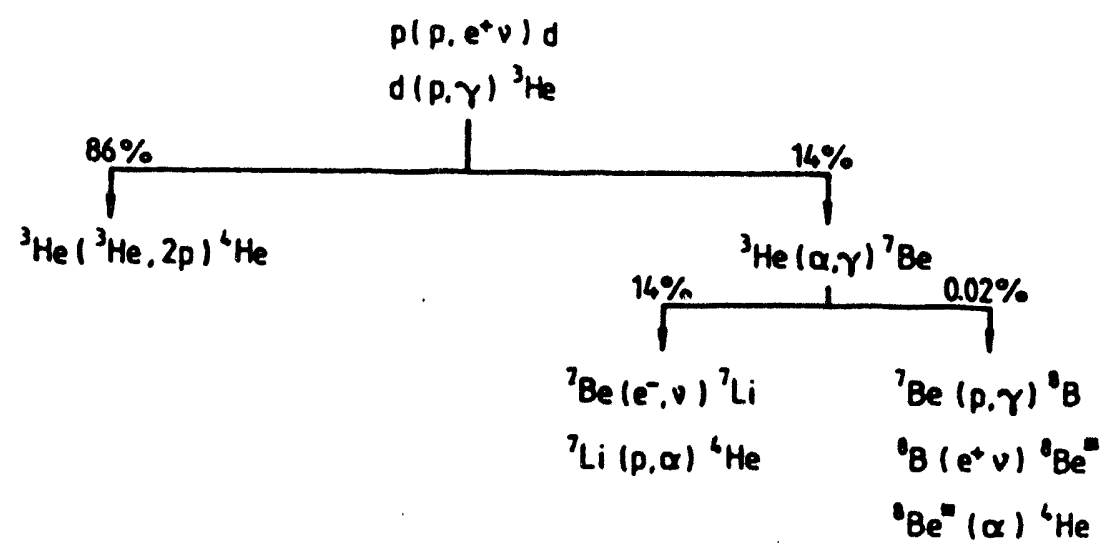

Figure 4 


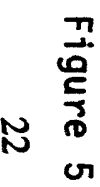

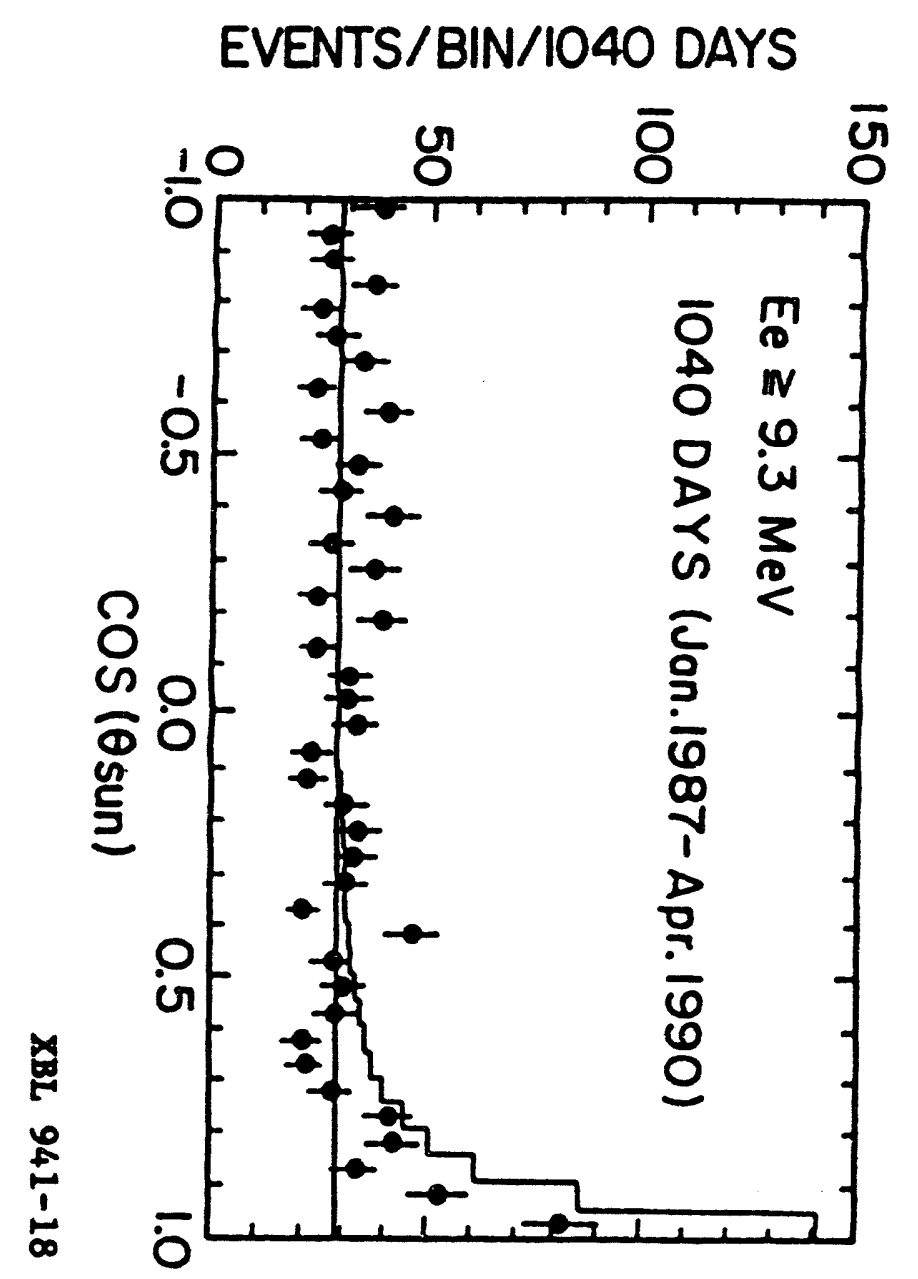


FIRST_STEP: $\quad \alpha+\alpha={ }^{8} \mathrm{Be}$

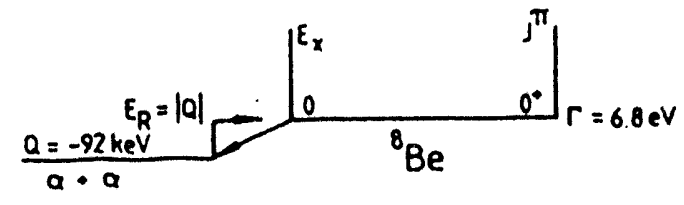

SECOND_SIEP_: $\quad{ }^{8} \mathrm{Be}(\alpha, \gamma)^{12 \mathrm{C}}$

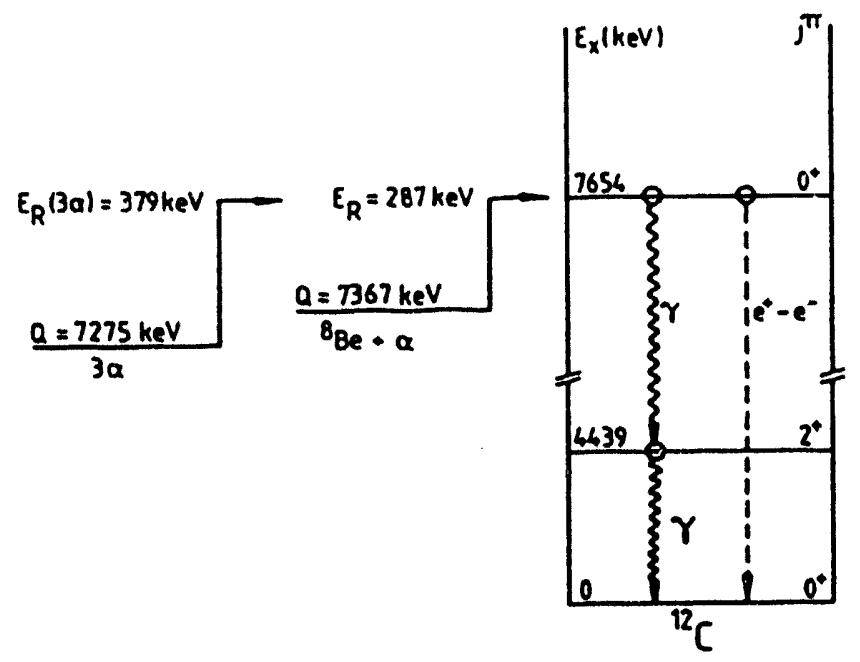

Figure 6 
INNER STRUCTURE OF A PRESUPERNOVA STAR

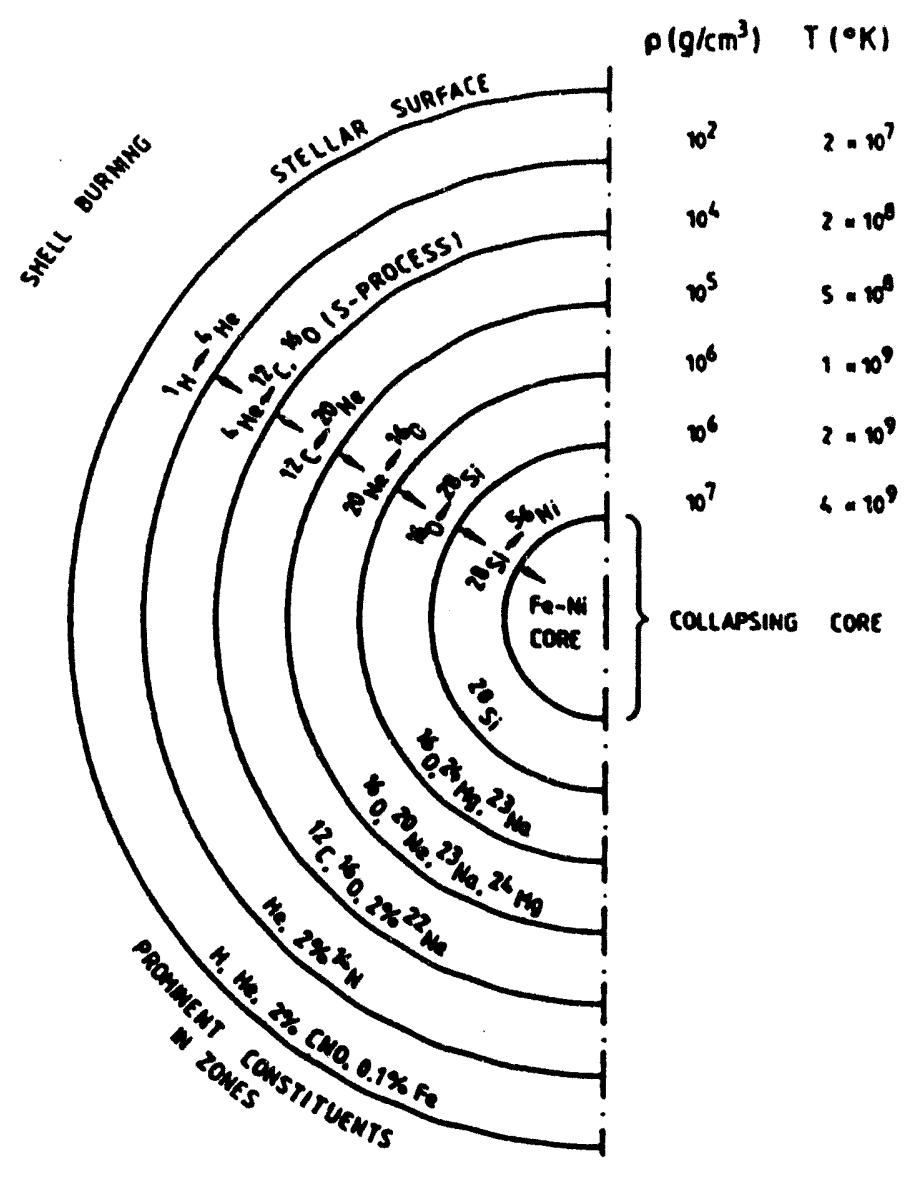

Figure 7 


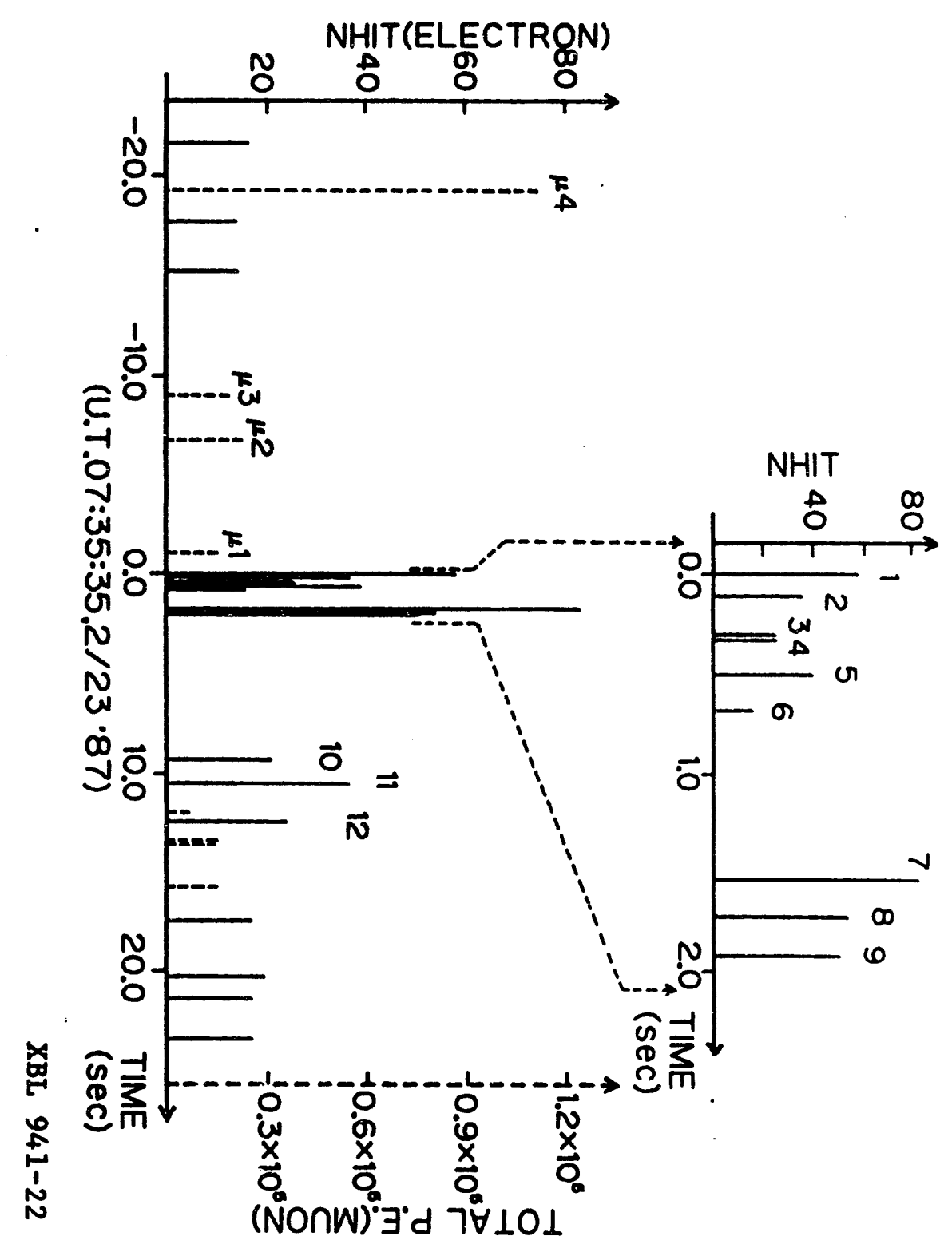




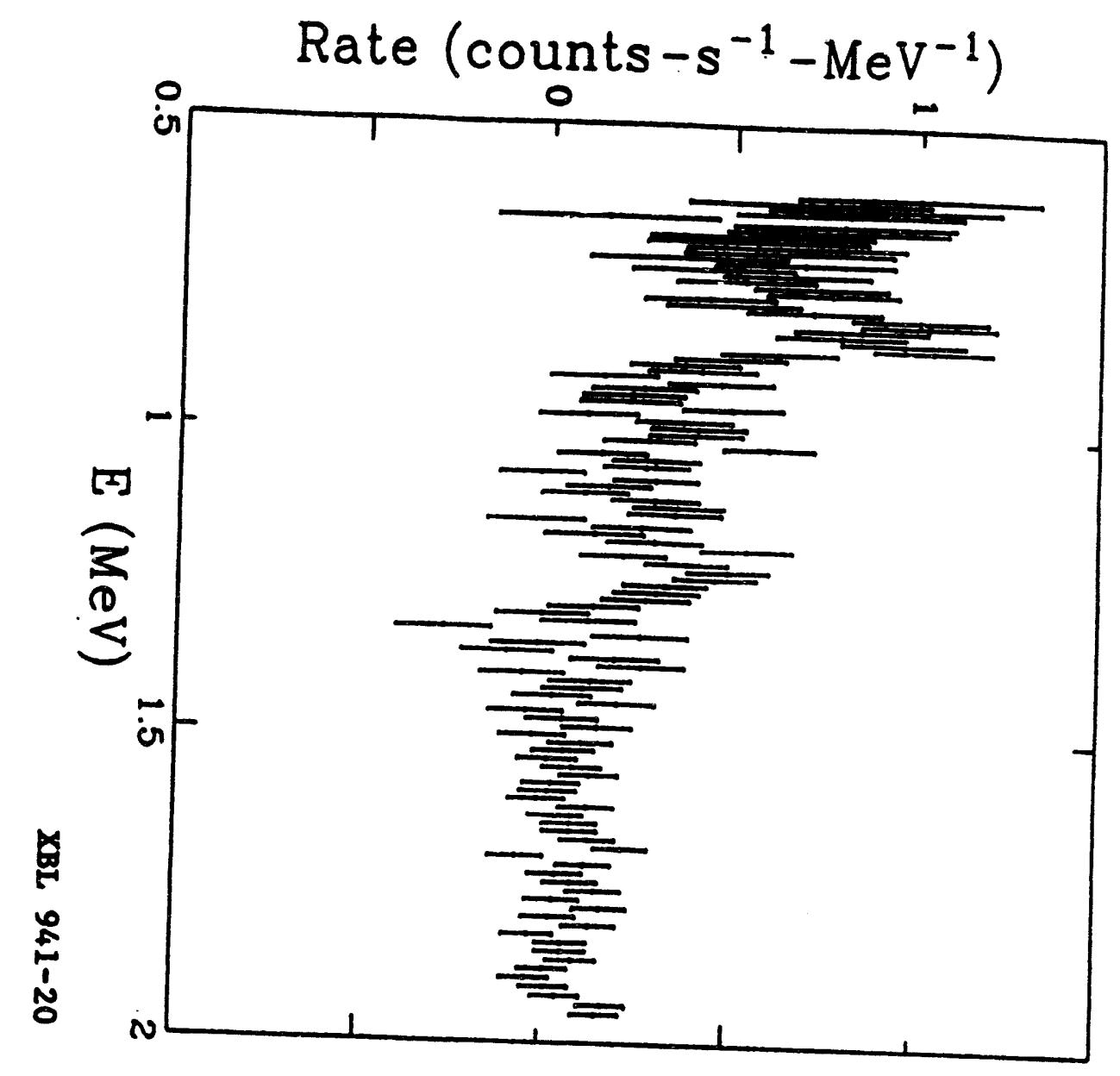




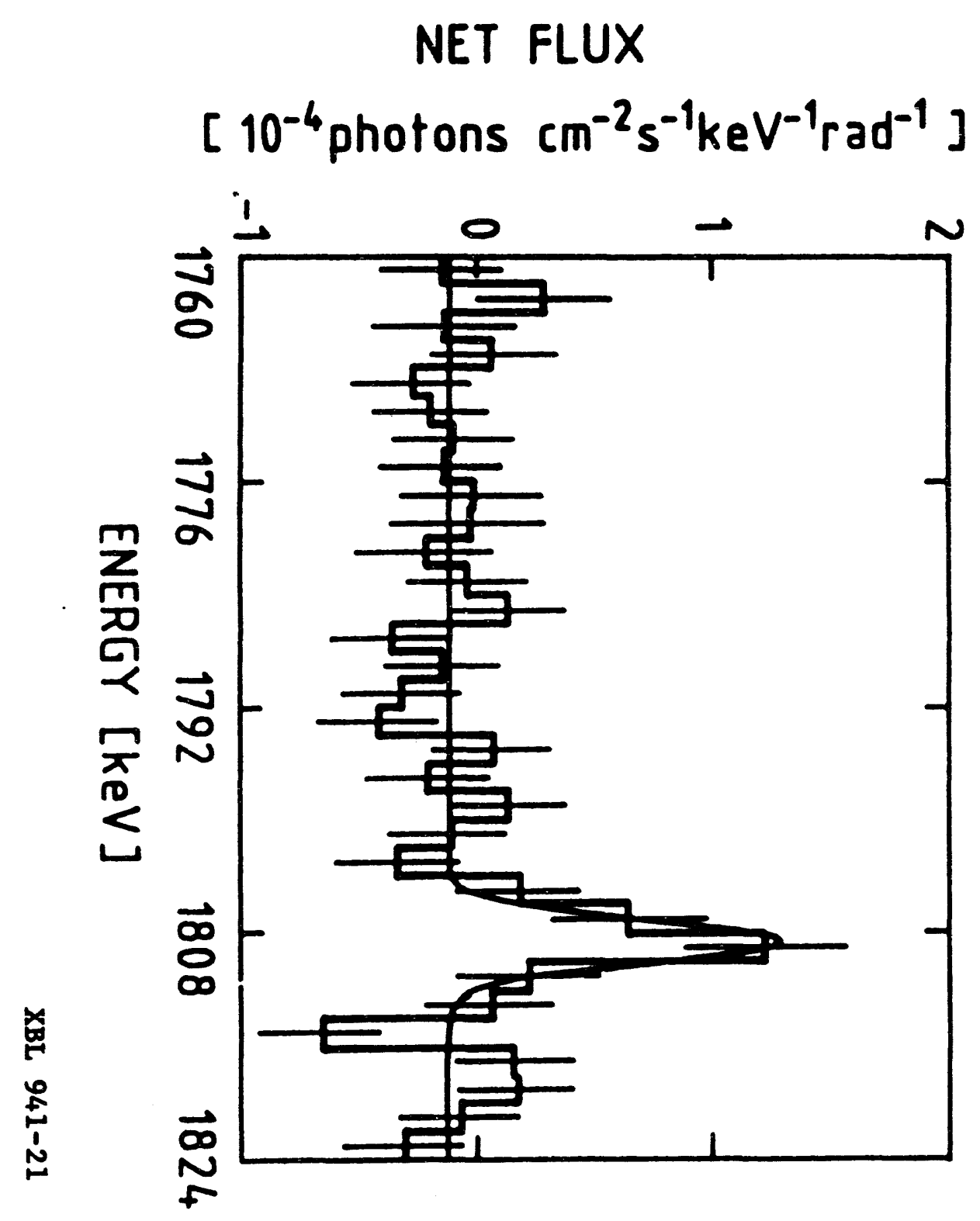




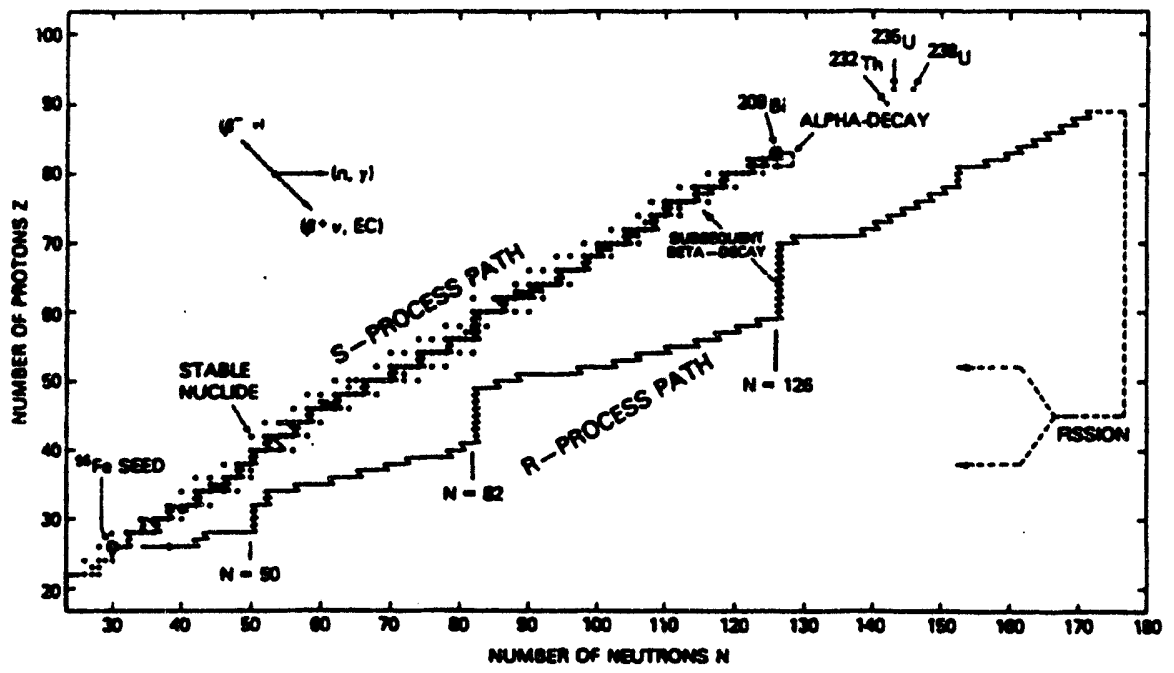

Figure 11 

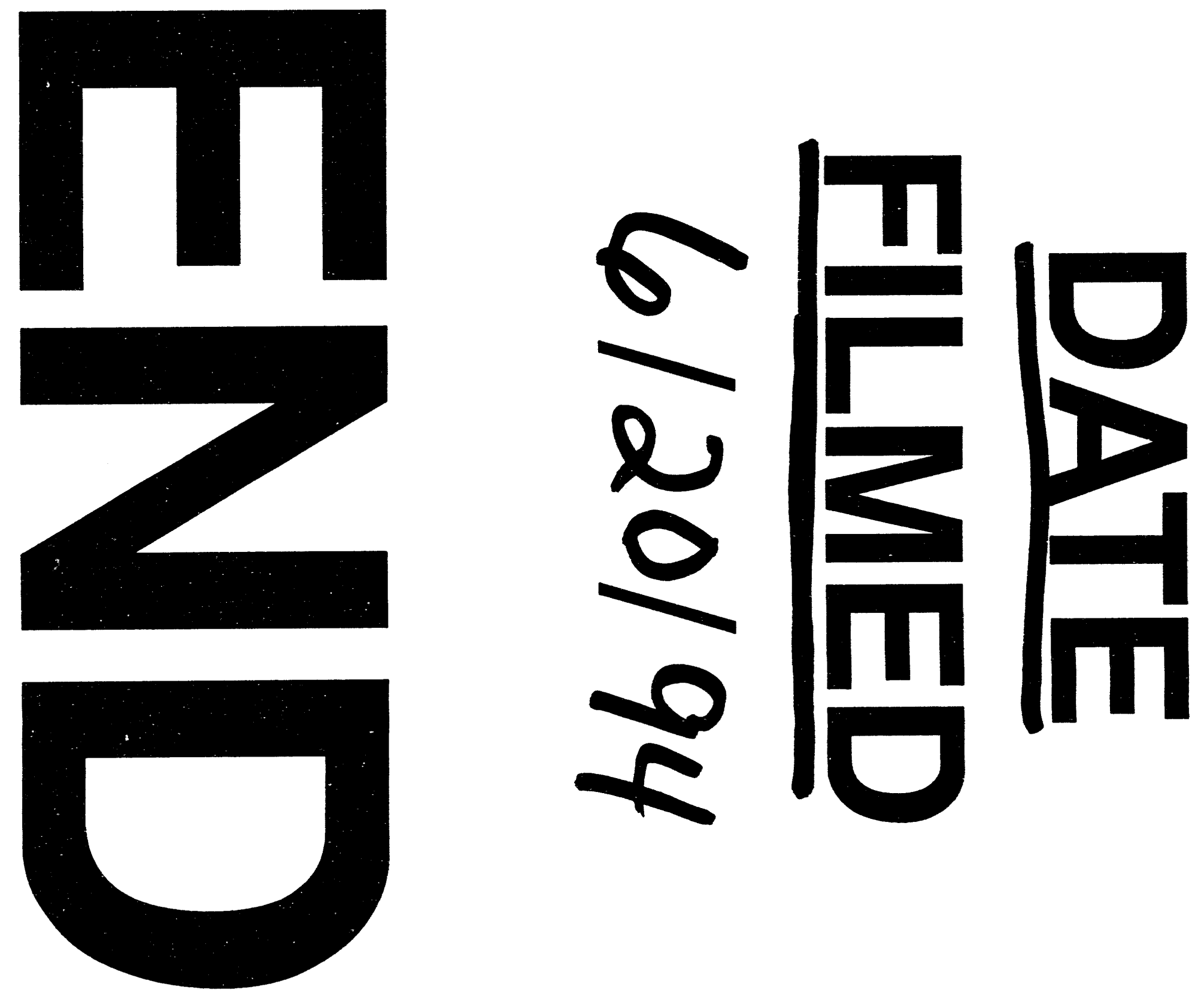
